\title{
Avaliação de xaropes contendo cloridrato de metoclopramida, pelo método de Bratton-Marshall
}

\author{
Beatriz Resende Freitas*, João Fernandes Magalhães \\ Departamento de Farmácia, Faculdade de Ciências Farmacêuticas, Universidade \\ de São Paulo
}

*Correspondência:

B. R. Freitas

R. Canário, 891 apto 146 - Moema 04521-004 São Paulo-SP-Brasil. E-mail: freitasbeatriz@uol.com.br
Atualmente, a maioria dos fármacos apresenta grupamento amínico. Estes quando associados a açúcares redutores ou a outros adjuvantes farmacêuticos contendo carbonila, freqüentemente produzem problemas de estabilidade, comprometendo a idoneidade do produto. A Reação de Maillard pode explicar tal ocorrência. Neste trabalho estudou-se o comportamento de xarope contendo amina aromática, tendo em vista a associação de açúcares e aminas, a Reação de Maillard e problemas de estabilidade. O protótipo escolhido foi o cloridrato de metoclopramida, benzamida com atividade farmacológica antiemética. Amostras dos xaropes de cloridrato de metoclopramida foram mantidas em estufa a $40{ }^{\circ} \mathrm{C}$ por seis meses. Em intervalos regulares de tempo alíquotas foram retiradas e submetidas à análise pelo método de Bratton-Marshall, seguida de leitura espectrofotométrica. Não houve grande variação no teor do cloridrato de metoclopramida em relação ao teor de açúcar, sendo que foram preparadas amostras padronizadas dos xaropes de cloridrato de metoclopramida em diferentes concentrações de açúcar. Houve diminuição do teor do cloridrato de metoclopramida, da ordem de 50\%, tanto para amostras padronizadas como para amostra comercial.
Unitermos

- Cloridrato de metoclopramida

- Reação de Maillard

- Reação de Bratton e Marshall

- Análise Quantitativa

- Espectrofotometria

- Amina aromática

\section{INTRODUÇÃO}

Conforme pode ser constatada em sua estrutura molecular, o cloridrato de metoclopramida é uma benzamida e seu nome químico é 4-amino-5-cloro- $N$-[2(dietilamino)-etil]-2-metoxibenzamida, sendo caracterizada como base fraca (Pitre, Strandi, 1987; USP, 1999; Merck, 2001). Exerce efeitos: antiemético, estimulante peristáltico e adjuvante do esvaziamento gastrintestinal. É bem absor- vida quando administrada por via oral. Sofre pequena transformação hepática e apresenta meia-vida plasmática de 2,6 a 6 horas em pacientes com função renal normal (Korolkovas, 1999). O cloridrato de metoclopramida é comumente utilizado na prevenção e alívio de náuseas e vômitos. Ademais, é empregado em associação à quimioterapia, em que fármacos, como a cisplatina e outros agentes citotóxicos, são altamente eméticos (Jong et al., 1987). 


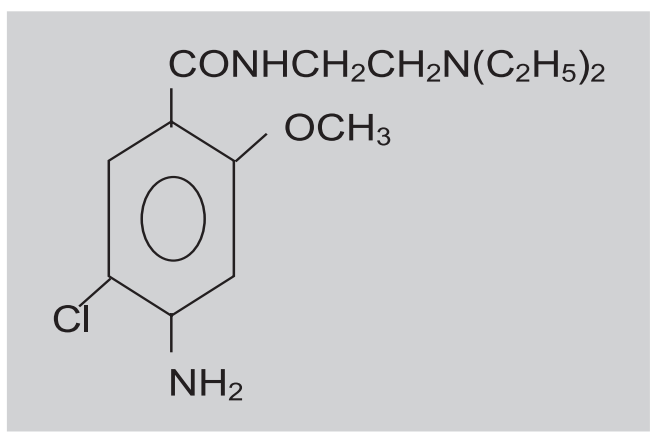

FIGURA 1 - Fórmula estrutural do cloridrato de metoclopramida

A reação de Maillard foi evidenciada e estudada pela primeira vez, em 1912, por Louis Maillard, o qual evidenciou que o aquecimento de aminoácidos e açúcares redutores precedia a formação de um pigmento marrom, o que indicaria problemas de incompatibilidade e estabilidade. (Labuza et al., 1994).

Com relação a produtos farmacêuticos a reação de Maillard tem grande importância, uma vez que a maioria dos fármacos disponíveis no mercado atual tem em sua composição aminas como grupamento funcional e estas, quando associadas a açúcares redutores ou a outros adjuvantes farmacêuticos contendo carbonila, freqüentemente, podem desencadear reações químicas que ocorrem durante o período de estocagem, indicando incompatibilidade e gerando problemas de estabilidade (Kumar, Banker, 1994). Outras experiências constataram que, em várias formulações comerciais líquidas contendo aminas e açúcares, em adição a outros adjuvantes, apresentaram interações referentes à reação de Maillard. (El-Kheir et al, 1975; Deshpande, Shirolkar, 1987; El-Kheir et al, 1991; Metwally et al, 1991).

O trabalho pioneiro de Bratton e Marshall (1939) para doseamento de sulfanilamida consistiu em empregar a reação de diazotação, formando o sal de diazônio, seguida de reação de acoplamento com dicloridrato de $N$-1-naftiletilenodiamina, formando, assim, a solução colorida. A eficiência do método em questão despertou o interesse de outros cientistas, contribuindo para o grande número de trabalhos publicados com tal metodologia (Martins, Magalhães, 1972; Santoro et al., 1984 a; Davidson, 1988; Morrison, Boyd, 1996; Revanasiddappa, Manju, 2001; Fan et al., 2001).

O trabalho atual teve como objetivo estudar o comportamento de xaropes contendo a amina aromática, cloridrato de metoclopramida, tendo em vista a associação aminas e açúcares, pelo método de Bratton-Marshall.

\section{MATERIAL E MÉTODO}

\section{Preparação da amostra padronizada do xarope de cloridrato de metoclopramida}

Inicialmente, foi preparada uma solução de xarope simples com concentração de $85 \%$ de açúcar e uma solução-mãe do cloridrato de metoclopramida com $27,50 \mathrm{mg} / \mathrm{mL}$. A partir destas, foram preparadas quatro soluções padronizadas de xarope de cloridrato de metoclopramida com diferentes concentrações de açúcar, nas concentrações de $82 \%, 68 \%, 54 \%$ e $41 \%$ p/v. Em todas essas, a concentração do cloridrato de metoclopramida foi a mesma $1,1 \mathrm{mg} / \mathrm{mL}$, conforme mostra a Tabela I.

Desta forma, foram obtidas 5 soluções dos xaropes de cloridrato de metoclopramida em diferentes concentrações de açúcar e concentração do cloridrato de metoclopramida de $1,1 \mathrm{mg} / \mathrm{mL}$

\section{Preparação da solução padrão do cloridrato de metoclopramida}

Utilizando um padrão secundário de cloridrato de metoclopramida (Farmasa), com potência de $100,55 \%$, foi preparada uma solução do cloridrato de metoclopramida a $22,0 \mu \mathrm{g} / \mathrm{mL}$. Como solvente foi utilizado ácido clorídrico $0,1 \mathrm{M}$.

TABELA I - Quantidades de solução-mãe do cloridrato de metoclopramida, xarope simples e água destilada para preparação de xaropes com diversas concentrações de açúcar

\begin{tabular}{ccccc}
\hline $\begin{array}{c}\text { balão de } \\
\mathbf{2 5 0} \mathbf{~ m L}\end{array}$ & $\begin{array}{c}\text { solução-mãe do cloridrato } \\
\text { de metoclopramida } \\
\mathbf{2 7 , 5} \mathbf{~ m g / m L}(\mathbf{m L})\end{array}$ & $\begin{array}{c}\text { xarope simples } \\
(\mathbf{m L})\end{array}$ & $\begin{array}{c}\text { água qsp } \\
(\mathbf{m L} \mathbf{L})\end{array}$ & $\begin{array}{c}\text { concentração de } \\
\text { açúcar } \mathbf{( \% )} \mathbf{p} / \mathbf{v}\end{array}$ \\
\hline 1 & 10 & 240 & - & 82 \\
2 & 10 & 200 & 250 & 68 \\
3 & 10 & 160 & 250 & 54 \\
4 & 10 & 120 & 250 & 41 \\
\hline
\end{tabular}




\section{Padronização do método de Bratton-Marshall para análise do cloridrato de metoclopramida}

Em série de experimetos, amostras do padrão do cloridrato de metoclopramida a $22,0 \mu \mathrm{g} / \mathrm{mL}$ foram submetidas a variações dos fatores interferentes potenciais. As análises foram feitas através do método de BrattonMarshall. A análise quantitativa foi realizada por espectrofotometria na região do visível, utilizando espectrofotômetro Hitachi U-2000, cubetas (Quartzo Suprasil ${ }^{\circledR}$ ) tipo 100-Q3. Uma alíquota de 5,0 mL da solução padrão do cloridrato de metoclopramida a $22,0 \mu \mathrm{g} / \mathrm{mL}$ foi transferida para balão volumétrico de $25 \mathrm{~mL}$, o qual foi acondicionado em banho de gelo. Esperou-se cinco minutos, iniciando a reação de diazotação com a adição de $1,0 \mathrm{~mL}$ de nitrito de sódio $(0,1 \%)$. Após dois minutos, foi adicionado $1,0 \mathrm{~mL}$ de sulfamato de amônio $(0,5 \%) \mathrm{e}$, finalmente, após mais dois minutos adicionou-se $1,0 \mathrm{~mL}$ de dicloridrato de $N$-1-naftil-etilenodiamina $(0,1 \%)$. Para estudos referentes à variação dos reagentes, fixou-se a quantidade dos demais em $1,0 \mathrm{~mL}$, variando a quantidade do reagente em estudo em 0,$5 ; 1,0 ; 1,5 ; 2,0$ e $2,5 \mathrm{~mL}$. A quantidade da solução do padrão do cloridrato de metoclopramida utilizada foi $5,0 \mathrm{~mL}$. Ao final da reação, o volume dos balões foi completado com ácido clorídrico $0,1 \mathrm{M}$. As absorvâncias foram determinadas a $537 \mathrm{~nm}$ usando-se ácido clorídrico $0,1 \mathrm{M}$ como branco. No estudo da variação da concentração do cloridrato de metoclopramida, mantendo o mesmo procedimento, foram utilizados 2, $0 ; 4,0 ; 5,0 ; 6,0$ e 8,0 mL da solução padrão do fármaco a $22,0 \mu \mathrm{g} / \mathrm{mL}$.

As mesmas soluções finais da experiência precedente, variação da concentração do cloridrato de metoclopramida, em que foram usados 2,$0 ; 4,0 ; 5,0 ; 6,0$; e $8,0 \mathrm{~mL}$ da solução padrão do cloridrato de metoclopramida a $22,0 \mu \mathrm{g} / \mathrm{mL}$, foram utilizadas para a determinação dos espectros de absorção. A leitura espectrofotométrica foi efetuada após a reação de Bratton-Marshall, realizada em balões de $25 \mathrm{~mL}$, obedecendo sistematicamente o procedimento descrito anteriormente e as soluções resultantes foram submetidas a uma faixa de leitura para determinação das absorvâncias de $440 \mathrm{~nm}$ a $600 \mathrm{~nm}$.

\section{Construção da curva de Ringbom e reta de calibração}

À partir das variáveis padronizadas utilizou-se uma solução padrão do cloridrato de metoclopramida a $22,0 \mu \mathrm{g} / \mathrm{mL}$, para construção da curva de Ringbom e da reta de calibração. As transmitâncias e absorvâncias foram determinadas a $537 \mathrm{~nm}$. Na construção da curva de Ringbom utilizaram-se 18 soluções com concentrações finais de 1,32;
1,$76 ; 2,20 ; 2,64 ; 3,08 ; 3,52 ; 3,96 ; 4,40 ; 5,28 ; 6,16 ; 7,04 ; 7,92$; 8,$80 ; 9,24 ; 9,68 ; 10,17 ; 10,56 ; 11,0 \mu \mathrm{g} / \mathrm{mL}$. Já na construção da reta de calibração foram utilizadas 10 soluções com concentrações finais de 1,$76 ; 2,64 ; 3,52 ; 4,40 ; 5,28 ; 6,16$; 7,$04 ; 7,92 ; 8,80 ; 9,68 \mu \mathrm{g} / \mathrm{mL}$.

\section{Validação do método analítico}

O método analítico foi validado segundo os critérios da Farmacopéia Americana 27a edição (USP, 2004). Foram avaliados os seguintes parâmetros: exatidão, precisão, linearidade, especificidade, limite de detecção e limite de quantificação.

A precisão e a exatidão foram determinadas realizando-se dez determinações espectrofotométricas a partir de 5,0 mL da amostra padronizada do xarope (matriz analítica) a $22,0 \mu \mathrm{g} / \mathrm{mL}$ e mais três determinações a partir de 5,0 mL da solução padrão a $22,0 \mu \mathrm{g} / \mathrm{mL}$. Submetidas à reação de Bratton-Marshall. Como branco utilizou-se ácido clorídrico $0,1 \mathrm{M}$.

A linearidade foi avaliada pelo método dos mínimos quadrados, com cinco concentrações diferentes referentes a concentrações de $50 \%, 75 \%, 100 \%, 125 \%$ e $150 \%$ do valor teórico do cloridrato de metoclopramida. O teste foi realizado em 3 triplicatas para cada análise.

Os limites de detecção e de quantificação foram determinados empregando a matriz analítica $(22,0 \mu \mathrm{g} / \mathrm{mL})$ e a solução padrão de metoclopramida a $22,0 \mu \mathrm{g} / \mathrm{mL}$. As absorvâncias foram efetuadas em três níveis de concentração de metoclopramida partindo-se de $0,5 \mathrm{~mL}, 1,0 \mathrm{~mL}$ e $1,5 \mathrm{~mL}$ da matriz analítica em cinco replicatas para cada nível.

\section{Avaliação da estabilidade das amostras padronizadas e da amostra comercial do xarope de cloridrato de metoclopramida}

As amostras padronizadas preparadas com diferentes concentrações de açúcar $(82 \%, 68 \%, 54 \%, 41 \%)$ e concentração de cloridrato de metoclopramida a $1,1 \mathrm{mg} / \mathrm{mL}$ (Item 2.1), assim como a amostra comercial do xarope de cloridrato de metoclopramida $(5 \mathrm{mg} / 5 \mathrm{~mL})$ foram mantidas em estufa, regulada para $40{ }^{\circ} \mathrm{C}$ durante seis meses. A intervalos regulares de tempo, a cada 30 dias, foram transferidos para balões volumétricos de $100 \mathrm{~mL}$ alíquotas de $10 \mathrm{~mL}$ das amostras padronizadas do xarope e da amostra comercial e os volumes foram completados com ácido clorídrico $0,1 \mathrm{M}$; de cada primeira diluição, $10 \mathrm{~mL}$ foram transferidos para balões volumétricos de $50 \mathrm{~mL}$, completando o volume com ácido clorídrico $0,1 \mathrm{M}$, desta forma preparando as soluções de análise com con- 
centração de $22,0 \mu \mathrm{g} / \mathrm{mL}$ do cloridrato de metoclopramida. Seqüencialmente 5,0 $\mathrm{ml}$ de cada última diluição (solução de análise a 22,0 $\mu \mathrm{g} / \mathrm{mL}$ ) foram submetidas à reação de Bratton e Marshall para análise quantitativa do princípio ativo por espectro-fotometria.

\section{RESULTADOS E DISCUSSÃO}

No estudo da variação dos fatores interferentes potenciais, para padronização da reação de Bratton-Marshall, a eventual influência de fatores interferentes, como a quantidade dos reagentes, foi descartada em face da pequena variação das absorvâncias obtidas nos experimentos. Portanto, a padronização do método foi realizada segundo a facilidade de manipulação, prevenindo-se, desta forma, erros de manipulação. Foi adotado $1,0 \mathrm{~mL}$ para todos os reagentes (nitrito de sódio, sulfamato de amônio e dicloridrato de $N$-1-naftiletilenodiamina), devido à facilidade de pipetagem. Também ficou evidenciada a relação proporcional entre os valores das absorvâncias e das concentrações, observada nos espectros de absorção em função da concentração do cloridrato de metoclopramida (Figura 2).

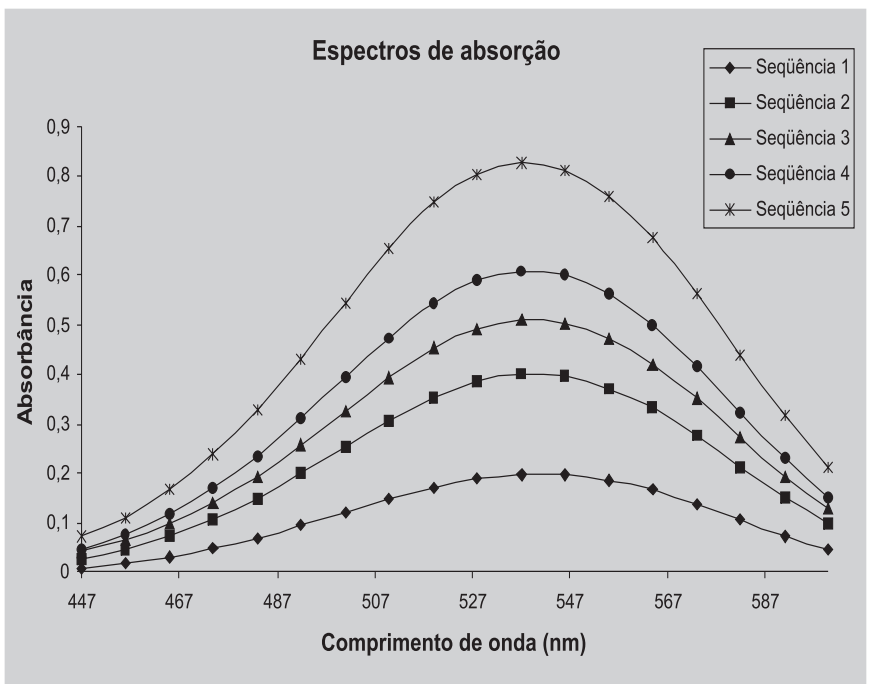

FIGURA 2 - Espectros de absorção em função da concentração do cloridrato de metoclopramida obtidos através de leitura espectrofotométrica em uma faixa de comprimento de onda de $440 \mathrm{~nm}$ a $600 \mathrm{~nm}$, após reação de Bratton-Marshall Seqüências 1,2,3,4 e 5 contendo 44,0 $\mu \mathrm{g}$; $88,0 \mu \mathrm{g} ; 110,0 \mu \mathrm{g} ; 132,0 \mu \mathrm{g}$ e $176 \mu \mathrm{g}$ do cloridrato de metoclopramida em ácido clorídrico $0,1 \mathrm{M}$, respectivamente.

A partir dos resultados dos espectros de absorção observados na Figura 2, ficou evidenciada a relação proporcional entre os valores de absorvâncias e das concen- trações. Mesmo assim, a curva de Ringbom foi construída, para consolidação dos resultados e estudo em uma larga faixa de concentração do cloridrato de metoclopramida. Houve resposta proporcional dos valores de concentração do ativo e absorvância, quando traçada a curva de Ringbom visualizando a curva sigmóide e consolidando a proporcionalidade apresentada.

A confirmação da adequada relação entre absorvância e concentração do cloridrato de metoclopramida, no método de Bratton-Marshall, pode ser observada na construção da reta de calibração, que apresenta ótima inclinação, garantindo a proporcionalidade entre as citadas variáveis, dados confirmados através da análise do valor do coeficiente de correlação (r), próximo de um $(0,9995)$, e do intercepto (b), próximo de zero $(0,0125)$, da reta de calibração, em que a equação da reta obtida foi $(\mathrm{y}=0,1185 \mathrm{x}+0,0125)$. Estes resultados assemelham-se àqueles obtidos na maioria dos casos com emprego deste método quando se trabalha com uma amina primária aromática isolada.

Do ponto de vista prático, e tratando-se de um método não cromatográfico, poder-se-iam considerar apenas os itens mais importantes na validação. De acordo com Brittain (1998), a validação de um método analítico é definida como sendo um processo através do qual estudos de laboratório são utilizados para garantirem que o método em questão atenda às exigências desejadas. No caso do presente trabalho, a determinação da exatidão, da precisão e da linearidade seria suficiente para os objetivos da pesquisa. Entretanto, o estudo foi estendido para outros parâmetros.

Na validação da metodologia analítica, a exatidão e a precisão - principais avaliações do processo de validação de métodos analíticos - apresentaram resultados muito satisfatórios. Obteve-se exatidão de 100,45\% e Desvio Padrão Relativo (DPR) de $0,32 \%$ referente à análise de dez replicatas da solução de análise $(22,0 \mu \mathrm{g} / \mathrm{mL})$ proveniente da diluição da amostra padronizada do xarope de cloridrato de metoclopramida a $1,1 \mathrm{mg} / \mathrm{mL}$ (Item 2.6). A linearidade foi determinada segundo Pasteelnick (1993), no que se refere à relação entre as concentrações teóricas e experimentais, sendo que os cinco valores experimentais são as médias das triplicadas obtidas pelo método padronizado referente às concentrações de $50 \%, 75 \%$, $100 \%, 125 \%$ e $150 \%$ da solução de análise da amostra do xarope de cloridrato de metoclopramida. Obteve-se coeficiente de correlação $(r=0,9998)$ e intercepto $(b=0,0044)$ satisfatórios. O limite de detecção encontrado foi de (LD $=0,126 \mu \mathrm{g} / \mathrm{mL}$ ) e o limite de quantificação (LQ) foi de $0,420 \mu \mathrm{g} / \mathrm{mL}$.

Os resultados referentes às análises das amostras 
dos xaropes de cloridrato de metoclopramida, submetidos à temperatura de $40^{\circ} \mathrm{C}$ por seis meses, estão na Tabela II.

Os resultados das análises sistemáticas realizadas com as amostras padronizadas e a amostra comercial do xarope, submetidas à $40^{\circ} \mathrm{C}$ por seis meses, demonstraram que há gradual decaimento do teor do cloridrato de metoclopramida, em todas as amostras. Tudo indica que a reação de Maillard seja a responsável pela interação química entre o cloridrato de metoclopramida e a sacarose do xarope.

A ocorrência de interação química entre açúcares redutores e aminas primárias e secundárias é fato já bastante conhecido (Castelo, 1962; Blaug, Huang, 1972; Blaug, Huang, 1973; Toledo, Bobbio, 1981; Tressler, 1985; Wirth, 1998). Como estas interações acontecem intensamente, mesmo com amostras no estado sólido e à temperatura ambiente, é lícito esperar que no estado líquido e a $40{ }^{\circ} \mathrm{C}$ a reação química entre as espécies envolvidas seja favorecida.

Neste estudo de estabilidade dos xaropes de cloridrato de metoclopramida preparados com diferentes concentrações de açúcar ficou evidenciado que os teores do cloridrato de metoclopramida diminuem mais rapidamente no início da estocagem, havendo tendência posterior para estabilização. Mesmo assim, durante todo o tempo de observação da experiência houve decréscimo da concentração, o que está demonstrado na Tabela II.

É interessante ressaltar que a variação na concentração de sacarose, pelo menos nos níveis em que foram estudados, não influenciou na velocidade, nem na intensidade de decaimento dos teores do cloridrato de metoclopramida. De fato, a diminuição ocorreu da mesma maneira, nas quatro amostras padronizadas estudadas (Tabela II).

A princípio, as experiências foram realizadas à temperatura ambiente. Entretanto, como poderiam ocorrer grandes variações ambientais no período de seis meses, previstos para observação da estabilidade, foi preferível evitar o risco da influência referente a tais variações térmicas. A escolha de $40{ }^{\circ} \mathrm{C}$, garantidos pela estocagem em estufa provida de termostato, não obedeceu a critérios préestabelecidos. O nível de temperatura escolhido está compatível com o clima tropical, igual ou um pouco acima da maioria das regiões do Brasil, sem o risco de afetar o produto do ponto de vista galênico. Temperaturas mais elevadas poderiam dificultar a parte analítica, uma vez que outras reações de decomposição poderiam ocorrer (Santoro et al., 1984 b). Neste caso, os resultados do doseamento poderiam ser influenciados de maneira incontrolável, como também poderiam gerar situações que não acontecem à temperatura ambiente.

De qualquer maneira, os resultados da avaliação servem para indicar o grau de estabilidade química de xaropes de cloridrato de metoclopramida, estocados por período de tempo relativamente curto, e para estudar possíveis conseqüências práticas. Fica evidente que formas farmacêuticas líquidas de cloridrato de metoclopramida não devem ser veiculadas em xaropes.

\section{CONCLUSÕES}

1 - Amostras padronizadas do cloridrato de metoclopramida, na forma de xarope, podem ser analisadas pelo método espectrofotométrico, usando-se a reação de Bratton-Marshall, desde que padronizado e validado para esta finalidade;

2 - Quando amostras padronizadas do cloridrato de metoclopramida, na forma de xarope contendo diferentes concentrações de sacarose, foram estocadas em estufa a $40{ }^{\circ} \mathrm{C}$ por seis meses, houve decomposição do fármaco e

TABELA II - Teor das análises periódicas de amostras padronizadas do xarope de cloridrato de metoclopramida em diferentes concentrações de sacarose e de uma amostra comercial

\begin{tabular}{|c|c|c|c|c|c|}
\hline \multirow{3}{*}{$\begin{array}{c}\text { Tempo } \\
\text { (dias) }\end{array}$} & \multicolumn{5}{|c|}{ Teor do cloridrato de metoclopramida encontrado $(\mathrm{mg} / \mathrm{mL})$} \\
\hline & \multicolumn{4}{|c|}{ Amostras padronizadas em concentração de sacarose } & \multirow{2}{*}{$\begin{array}{l}\text { Amostra } \\
\text { comercial }\end{array}$} \\
\hline & $41 \%$ & $54 \%$ & $68 \%$ & $82 \%$ & \\
\hline 0 & 21,958 & 22,460 & 21,749 & 21,665 & 18,821 \\
\hline 30 & 20,076 & 19,741 & 19,658 & 19,449 & 15,057 \\
\hline 60 & 16,395 & 16,186 & 16,312 & 16,939 & 14,011 \\
\hline 90 & 14,221 & 14,639 & 15,057 & 14,639 & 12,548 \\
\hline 120 & 13,593 & 13,886 & 13,259 & 13,802 & 11,920 \\
\hline 150 & 13,175 & 12,840 & 12,757 & 12,338 & 10,373 \\
\hline 180 & 11,711 & 11,502 & 12,213 & 11,293 & 9,411 \\
\hline
\end{tabular}

Análises mensais pelo método de Bratton-Marshall em baixa temperatura $\left(0-2{ }^{\circ} \mathrm{C}\right)$, seguida de leitura espectrofotométrica a $537 \mathrm{~nm}$ (O valor da leitura em absorvância do padrão foi de 0,526$)$. 
o teor caiu cerca de $50 \%$ em relação ao valor inicial, independente das concentrações de açúcar usadas na preparação das amostras;

3 - A decomposição do fármaco foi observada tanto nas amostras padronizadas como na amostra comercial submetida à análise, comprovando a importância do trabalho para alertar indústrias farmacêuticas quanto a problemas de estabilidade em relação às associações que podem favorecer a reação de Maillard.

\section{ABSTRACT}

\section{Evaluation of metoclopramide syrups by Bratton-Marshall method}

Nowadays, most of the drugs have amine group in their structure. These drugs, when associated to sugar reducers, or other carbonyl excipients frequently produce dark stains or fading. The Maillard reaction can explain such occurrence. In this work, we have studied the behavior of syrups containing aromatic amines. It is known that association of sugars and amines can generate problems of stability. The chosen prototype was the metoclopramide hydrochloride, a benzamide, with anti-emetic pharmacological activity. Samples of the metoclopramide syrups were maintained in stove at $40{ }^{\circ} \mathrm{C}$ for six months. In regular time intervals aliquots were removed and submitted to quantitative determination by the Bratton-Marshall's method, followed by a spectrophotometric determination. There was not great variation of the metoclopramide level in relation to sugar level, once metoclopramide samples were prepared in different sugar levels. There was a decrease of the metoclopramide level, over 50\%, for commercial and standardized samples, after a period in stove

UNITERMS: Metoclopramide hydrochloride. Maillard reaction. Bratton-Marshall reaction. Quantitative analysis. Spectrophotometry. Aromatic amine.

\section{REFERÊNCIAS BIBLIOGRÁFICAS}

BLAUG, S.M.; HUANG, W.-T. Interation of dextroamphetamine sulfate with spray-dried lactose. $J$. Pharm. Sci., New York, v.61, n.11, p.1770-1775, 1972.

BLAUG, S.M.; HUANG, W.T. Interaction of dextroamphetamine sulfate with dextrose in solution. $J$. Pharm. Sci., New York, v.62, p.653-655, 1973.
BRATTON, A.C.; MARSHALL Jr., E.K. A new coupling component for sulfanil-amine determination. J. Biol. Chem., Bethesda, v.128, p.537-550, 1939.

BRITTAIN, G.H. Validação de métodos não cromatográficos. Pharm. Technol., São Paulo, v. 30, n. 3, p.4-9, 1998.

CASTELO, R.A.; MATTOCKS, A.M. Discoloration of tablets containing amines and lactose. J. Pharm. Sci., New York, v.51, n.2, p.106-108, 1962.

DAVIDSON, A.G. Ultraviolet-visible absorption spectrophotometry. In: BECKETT, A.H., STENLAKS, S.B., (Eds.). Practical pharmaceutical chemistry. 4.ed. London: Athlone Press, 1988. v.2, p.301-302.

DESHPANDE, S.G.; SHIROLKAR, S. Stability of bromexine hydrochloride in liquid oral formulations. East. Pharm., New Delhi, v. 30, n. 357, p.135-138, 1987.

EL-KHEIR, A.A.; SALEM, F.B.; AHMAD, A.K.S.; AMER, M.M. Browning reactions in pharmaceutical preparations containing liquid glucose. Egypt. J. Pharm. Sci., Cairo, v.61, p.219-231, 1975.

EL-KHEIR, A.A.; EL-BOLKINEY, M.N.; EL-SAYED, W.; METWALLY, M.M.F. Study for factores affecting browning reaction in stored cough mixtures. Egypt. J. Pharm. Sci., Cairo, v.32, p.175-180, 1991.

FAN,J.; CHEN, Y.H.; YE, C.L.; FENG, S.L. Flow injection spectrophotometric determination of metoclopramide. Chinese J. Anal. Chem., Changchun, v.29, n.2, p.216218, 2001.

JONG, A.P.; WITTEBROOD, A.J.; CHÂNTINIER, W.M.D.; BRON, J. Liquid chromatographic analysis of alizapride and metoclopramide in human plasma and urine using solid phase extraction. J. Chromatogr., Amsterdam, v.419, p.233-242, 1987.

KOROLKOVAS, A. Dicionário terapêutico Guanabara. 6.ed. Rio de Janeiro: Guanabara Koogan, 1999. p.10.2710.28 .

KUMAR, V.; BANKER, G.S. Maillard reaction and drug stability. In: LABUZA, T.P.; REINECCIUS, G.A.; MONNIER, V.; O'BRIEN, J.; BAYNES, J., (Eds.). Maillard reactions in chemistry, food, and health. Cambrige: Royal Society of Chemistry, 1994. 440p. 
LABUZA, T.P.; REINECCIUS, G.A.; MONNIER, V.; O'BRIEN, J.; BAYNES, J., Maillard reactions in chemistry, food, and health. Cambrige: Royal Society of Chemistry, 1994. 440p.

MARTINS, J.L.S.; MAGALHÃES, J.F. Contribuição à espectrofetometria de sulfanilamidas. Rev. Farm. Bioquím. Univ. São Paulo, São Paulo, v.10, n.2, p.167176, 1972.

MERCK index. 13.ed. New Jersey: Whitehouse Station, 2001.p.1094, 6164.

METWALLY, M.M.F.; EL-BOLKINEY, M.N.; ELSAYED, W.; EL-KHEIR, A.A. Identifications of the carbonyl compounds formed in stored cough mixtures. Egypt. J. Pharm. Sci., Cairo, v.32, p.787-796, 1991.

MORRISON, R.T.; BOYD, R.N. Química orgânica. 13.ed. Lisboa: Fundação Calouste Gulbenkian, 1996. p.923-999.

PASTEELNICK, L.A. Analytical methods validation. In: BERRY, I.R., NASH, R.A., eds. Pharmaceutical process validation. 2 ed. New York: Marcel Deckker, 1993. cap.13, p.411-428.

PITRE, D., STRANDI, R. Metoclopramide hydrochloride. Anal. Profiles Drug Subst., New York, v.16, p.327-360, 1987

REVANASIDDAPPA, H.D.; MANJU, B. A spectrophotometric method for the determination of metoclopramide $\mathrm{HCl}$ and dapsone. J. Pharm. Biomed. Anal., Amsterdam, v.25, n.3/4, p.631-637, 2001.
SANTORO, M.I.; SANTOS, M.M.; MAGALHÃES, J.F. Spectrophotometric determination of bromhexine hydrochloridre in pharmaceutical preparations. J. Assoc. Off. Anal. Chem., Washington, v.67, n.3, p.532-534, $1984 \mathrm{a}$.

SANTORO, M.I.R.M.; HACKMANN, E.R.M.; MAGALHÃES, J.F.; VERNENGO, M.J. Estudo cinético preliminar da decomposição da glicose em sais de reidratação oral. Rev. Farm. Bioquím. Univ. São Paulo. v.21, n.1, p.80-90, 1984 b.

TOLEDO, M.C.F.; BOBBIO, P.A. Maillard rection VII: browning of glucose with lysine, clycine and glutamic acid. An. Acad. Brasil. Ciênc., Rio de Janeiro, v.53, n.4, 1981.

TRESSLER, L.J. Medicine botlle caps. Pharm. J., London, v.235, p.99, 1985.

UNITED States Pharmacopeia. 27.ed. Rockville: United States Pharmacopeial Convention, 2004.p.1097-1099.

WIRTH, D.D.; BAERTSCHI, S.W.; JOHNSON, R.A.; MAPLE, S.R.; MILLER, M.S.; HALLENBECK, D.K.; GREGG, S.M. Maillard reaction of lactose and fluoxetine hydrochloride, a secondary amine. J. Pharm. Sci., New York, v.87, n.1, p.31-39, 1998.

Recebido para publicação em 6 de outubro de 2003 Aceito para publicação em 15 de junho de 2005 